\title{
Functional hyper-extension deficit of the knee and patellar dimple sign in patellofemoral pain syndrome. A preliminary study
}

\author{
Defne Kaya ${ }^{1}$ \\ Ertugrul Aksahin² \\ Mahmut Nedim Doral ${ }^{3}$
}

\footnotetext{
1 NP Physiotherapy and Rehabilitation Clinic \& Head of Department of Physiotherapy and Rehabilitation, Faculty of Health Sciences, Uskudar University, Istanbul, Turkey

2 Department of Orthopaedic and Traumatology, Medical Park Hospital, Ankara, Turkey

3 Department of Orthopaedic and Traumatology, Faculty of Medicine, Hacettepe University, Ankara, Turkey
}

Corresponding author:

Defne Kaya

NP Physiotherapy and Rehabilitation Clinic,

Department of Physiotherapy and Rehabilitation,

Faculty of Health Sciences

Uskudar University

Altunizade Mahallesi,

Haluk Türksoy Sk. 14

34662 Üsküdar, Istanbul, Turchia

E-mail: defne1976@gmail.com

\section{Summary}

Introduction: The aim of this study was to investigate the presence of two new signs in patellofemoral pain syndrome (PFPS).

Methods: Twenty-three patients with unilateral patellofemoral pain were participated.

The study was designed with each subject acting as their own internal control by using the unaffected limb for comparison. The pain level during squat \& stair up-down, functional hyper-knee-extension lack, absence of the patellar dimple sign, and $\mathrm{Ku}-$ jala patellofemoral score were measured in patients.

Results: All patients had an unclear and weak patellar dimple sign. Mean pain level during the squat was $7.13 \pm 2.54$. The functional hyper-knee-extension lack was $4.39 \pm 1.23^{\circ}$ in the affected side while was $3.91 \pm 1.37^{\circ}$ in the unaffected side.

Conclusion: This study is the first to demonstrate absence of the patellar dimple sign and functional hyperextension lack in patients with patellofemoral pain syndrome. It is suggested that future studies might look at the effect of functional hyperextension knee exercises and look at the longer term fol- low-up of these signs.

Level of evidence: IIlb.

KEY WORDS: patellar dimple sign, functional hyperextension, patellofemoral pain syndrome.

\section{Introduction}

Patellofemoral pain syndrome (PFPS) is characterised by anterior, retropatellar or peripatellar pain and its etiology is multifactorial including quadriceps weakness ${ }^{1,2}$, excessive flattening of the medial arch and instability of the forefoot influencing internal rotation of tibia, compensatory internal rotation of femur ${ }^{2,3}$, and consequent patellar malalignment ${ }^{4,5}$.

Although apprehension test, Bassett's sign, patellar glide test, Q-Angle, patellar tilt test, Quadriceps pull test, and J-sign should be used to evaluate the patellofemoral joint malalignment, the sensitivity/specificity-reliability/validity of these tests remains unclear ${ }^{6}$. Particularly, dynamic and static Q-Angle, a J-sign, and patellofemoral displacement have been described as clinical parameters and signs for patellofemoral pain syndrome while the reliability and validity of these tests are also unclear?.

Although several clinical tests to evaluate of the patellar malalignment have been described in patients with PFPS, the presence of functional hyperknee-extension lack and absence of the patellar dimple sign in this group of patients has not been investigated in the literature. Therefore, the aim of this study was to investigate the presence of functional hyperextension lack and patellar dimple sign in PFPS. Our hypothesis was that there would be patellar dimple sign consist of functional hyper-knee-extension lack in patients with PFPS.

\section{Materials and methods}

Institutional Review Board (IRB) approval and patients consent was obtained prior to conducting the investigation. All method complies with ethical standards of Muscle, Ligaments and Tendons Journal as previously described ${ }^{8}$. This was a case series study with each subject acting as their own internal control by using the unaffected limb.

Twenty-three female patients (age: $39 \pm 5$ years; height: $1.62 \pm 0.53 \mathrm{~m}$; weight: $78.4 \pm 10.6 \mathrm{~kg}$ ) with unilateral PFPS were referred from the orthopaedic departments. All measurements were done bilateral- 
ly. Patients were briefed about aims of the study, and the testing procedure prior to their participation. Written informed consent was obtained.

Patients were included in this study if the following criteria were fulfilled: the onset of pain was longer than six months, characteristic clinical signs (i.e., retropatellar pain, crepitation and pain in patellar grinding), and positive clinical tests (Clarke's sign ${ }^{9}$, active patellar grind test ${ }^{10}$, direct patellar compression ${ }^{10}$, palpation of the medial articular border of the patella10,11, and palpation of the lateral articular border of the patel$\mathrm{la}^{10,11}$ ) of the syndrome ages between $30-50$ years, no history or clinical evidence of patellofemoral dislocation, subluxation, or osteoarthritis, and no history of lower extremity surgery, absence of knee ligaments, bursae, menisci, and synovial plicae dysfunction in clinical examination, no cartilage, ligament, plicae, and meniscal lesion in MRI assessment. Positive MRI finding for patellofemoral syndrome was in all the patients.

\section{Pain}

An 11-point numeric rating scale was used to assess pain in $1 \mathrm{~cm}$ intervals, anchored on the left with the phrase "no pain" and on the right "worst imaginable pain"12. The patients rated their pain during performance of an aggravating activity (step down-up and step-down activities from a standard $20.3 \mathrm{~cm}$ gym bench; full squat) and pain on squat position. Results were approximated to the nearest millimetre.

\section{Functional hyper-knee-extension test}

Functional hyper-knee-extension was measured in the supine position and pillow under the heel with quadriceps isometric contraction (Fig. 1). A pillow under the heel helps to promote full straightening of the knee and is recommended. The long arms of the goniometer were placed along the long axis of the femur and the other along the tibia, respectively. For functional hyper-knee-extension, arms of the goniometer were moved neutral position (0) to full hyperextension with maximal volunteer isometric quadriceps contraction. A negative value indicated hyper-knee-extension while positive value indicated presence of the hyperknee-extension lack.

\section{Patellar dimple sign}

During the functional hyper-knee-extension test (pillow under the heel) and maximal volunteer isometric quadriceps contraction, patellar dimples as a smile icon occurred at the superior-medial corner of the patella in the unaffected side of the patients (Fig. 2). The patellar dimples in the unaffected side of the patients are clear and strong while patellar dimples in the affected side of the patients are unclear and weak.

\section{The functional level}

Kujala patellofemoral score was used to evaluate functional status of the patients. It is a knee specific self-report score to assess six activities which are as-

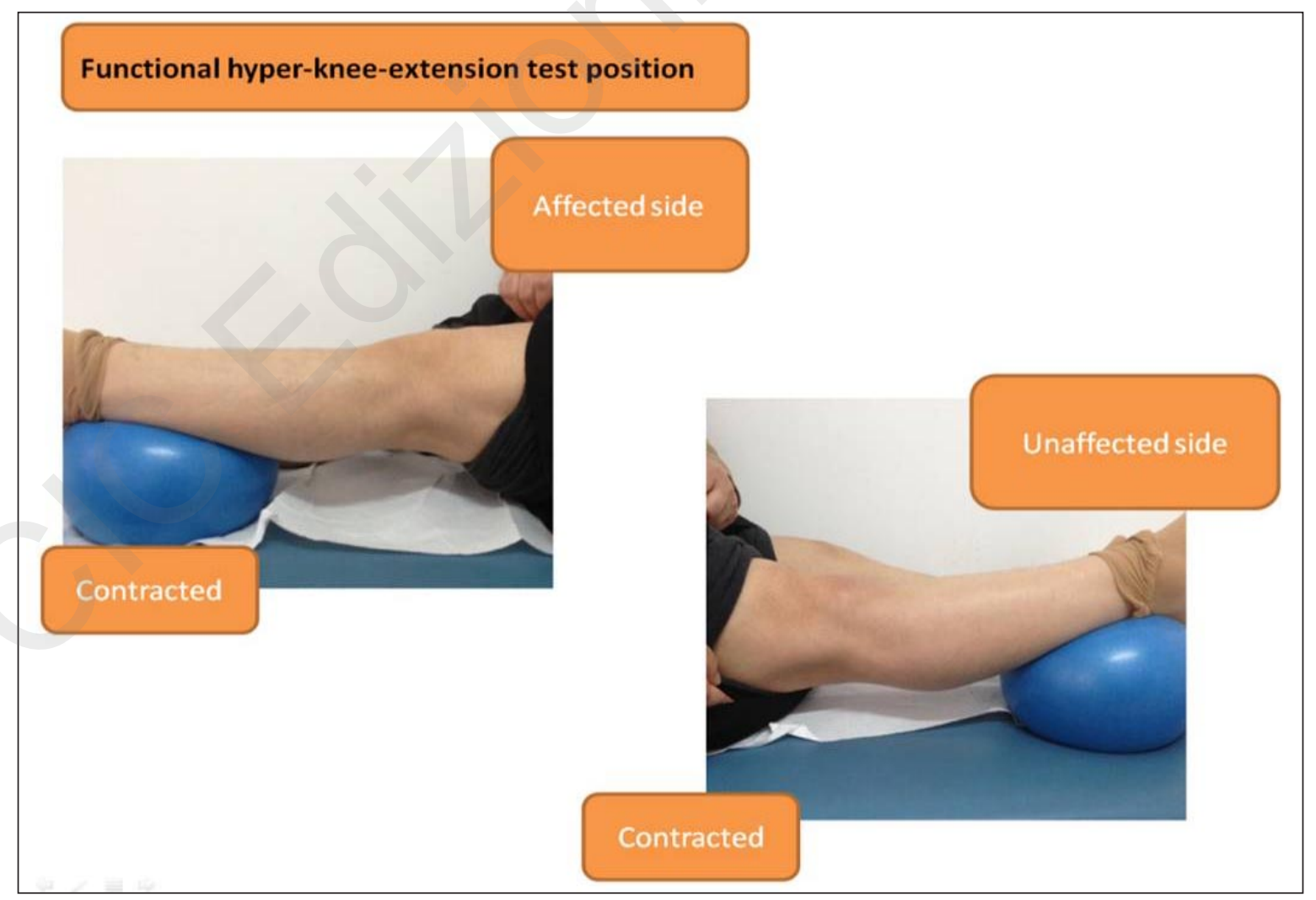

Figure 1. Position for the functional hyper-extension test. 


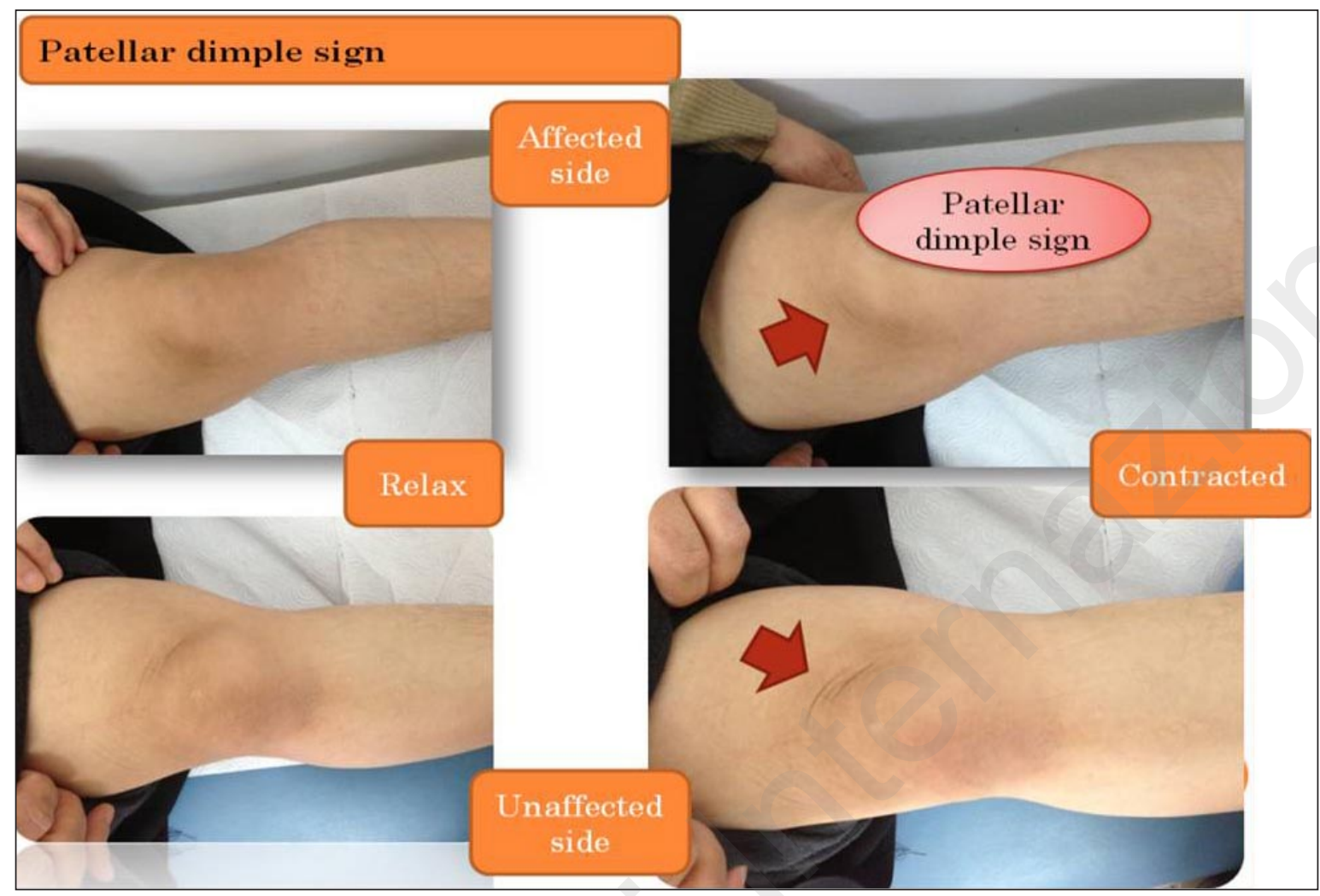

Figure 2. Patellar dimple sign.

sociated with patellofemoral pain syndrome (walking, running, jumping, climbing, stairs, squatting and sitting). The maximum total score is 100 , with higher scores indicate good functional level ${ }^{13}$.

\section{Statistical analysis}

All data were analysed with the Statistical Package for the Social Sciences version 14.0. Mean and standard deviations were collected.

\section{Results}

All measurements of 23 patients were completed. Average pain was $7.13 \pm 2.54$ during squat, $4.47 \pm 3.45$ during stairs-up, and $4.65 \pm 3.63$ during stairs-down.

The functional hyper-knee-extension was $+4.39 \pm 1.23$ $\left({ }^{\circ}\right)$ in the affected side while was $-3.91 \pm 1.37\left(^{\circ}\right)$ in the unaffected side. All patients had weak and unclear patellar dimple sign in the affected side. Kujala patellofemoral score average was $55.78 \pm 16.87$ (range, 21 to 76 ).

\section{Discussion}

The most important finding of the present preliminary study was the presence of the functional hyper-kneeextension lack and absence of the patellar dimple sign in patients with patellofemoral pain syndrome. This study was the first to describe the presence of functional hyper-knee-extension lack and absence of the patellar dimple sign in patients with PFPS.

Common causative factors in patellofemoral pain syndrome are lower extremity weakness, especially in the quadriceps muscles, malalignment of the lower extremity, foot deformity such as hallux valgus and increased subtalar pronation, tightness of the lateral retinaculum, iliotibial band, hamstring muscles and tensor fascia latae muscle 1,2,4,11-16. Correct patellar alignment may depend in part upon the balance of strength and neuromuscular activation timing between the vastus medialis obliquus (VMO) and vastus lateralis (VL) muscles. A VMO, VL imbalance is one proposed mechanism for abnormal patellar tracking ${ }^{14}$. Generalized quadriceps muscle weakness may result in patellar mal-position ${ }^{15}$, and altered VMO timing, suggesting that muscle length changes at activation onset will negatively influence is force producing capability ${ }^{17}$. Addition to these well-known etiological factors of the patellofemoral pain syndrome, we observed lack of functional hyper-knee-extension in the affected side of the patients with PFPS. In the present study, the mean functional hyper-knee-extension was +4 degree in the affected side while was -3 degree in the unaffected side. Seven degrees deficiency was found between the sides. The goniometric measurement was used to determine functional hyperknee-extension lack. The future studies are needed to evaluate functional hyper-knee-extension lack using motion and gait analysis system. Our findings 
here lead us to speculate that functional hyper-kneeextension lack should be one of the common etiological problems of the patellofemoral pain syndrome. The patellar mal-position, lower extremity mal-alignment, quadriceps timing problems, and abnormal patellar tracking may also cause knee range of motion deficits such as functional hyper-knee-extension lack. Additionally, our hypothesis was that there would be absence of the patellar dimple sign caused by functional hyper-knee-extension lack in patients with PFPS. The tendency of the flexion of the femur and tibia may cause the lack of the functional hyperknee-extension. The lack of the functional hyper-extension may preclude of the presence of the patellar dimples. The absence of the patellar dimple sign was found in the affected side of all patients in the present study. Clearly, a longitudinal study measuring the functional hyper-knee-extension lack would be required along with further biomechanical studies to find out presence of the functional hyper-knee-extension lack and relationship with absence of the patellar dimple sign in patients with PFPS.

The prime limitation of our study was that, was lack of biomechanical analysis to evaluate functional hyperextension of the knee. Another limitation is that we did not assess the efficacy of treatment. Another issue is that this study has been unable to prove cause and effect; in other words, does the pain cause functional hyper-knee-extension lack or does functional hyper-knee-extension lack bring about pain?

\section{Conclusion}

This study is the first to demonstrate the functional hyperextension lack and absence of the patellar dimple sign in female patients with patellofemoral pain syndrome. It is needed that future studies might investigate patellar dimple using leg extension test ${ }^{18}$, isokinetic test for muscle strength ${ }^{19}$, functional MRI (fMRI) for patellar position and patellar dimple sign during activities, movement analyses for the knee angles, and electromyographic (EMG) actions ${ }^{18}$ for vastus medialis and vastus lateralis during activities. It is also suggested that future studies might look at the effect of functional hyperextension knee exercises/techniques and look at the longer term follow-up of these patients.

\section{Conflict of interest}

Authors have no conflict of interest.

\section{References}

1. Miller JP, Croce RV. Analysis of Isokinetic and Closed Chain Movements for Hamstring Reciprocal Coactivation. Journal of Sport Rehabilitation. 2007;16:319-325.

2. Powers CM, Bolgla LA, Callaghan MJ, Collins N, Sheehan FT. Patellofemoral pain: proximal, distal, and local factors, 2nd In- ternational Research Retreat. J Orthop Sports Phys Ther. 2012;42:A1-54

3. Barton C, Levinger P, Crossley K, Webster K, Menz H. The relationship between rearfoot, tibial, and femoral kinematics in individuals with patellofemoral pain syndrome. J Orthop Sports Phys Ther. 2012;42:A32.

4. Derasari A, Brindle TJ, Alter KE, Sheehan FT. McConnell taping shifts the patella inferiorly in patients with patellofemora pain: a dynamic magnetic resonance imaging study. Phys Ther. 2010;90:411-419.

5. Syme G, Rowe P, Martin D, Daly G. Disability in patients with chronic patellofemoral pain syndrome: a randomised controlled trial of VMO selective training versus general quadriceps strengthening. Man Ther. 2009;14:252-263.

6. Smith TO, Davies L, O'Driscoll ML, Donell ST. An evaluation of the clinical tests and outcome measures used to assess patellar instability. Knee. 2008;15:255-262.

7. Sheehan FT, Derasari A, Fine KM, Brindle TJ, Alter KE. Q-angle and J-sign: indicative of maltracking subroups in patellofemoral pain. Clin Orthop Relat Res. 2010;468:266275.

8. Padulo J, Oliva F, Frizziero A, Maffulli N. Muscles, Ligaments and Tendons Journal - Basic principles and recommendations in clinical and field science research: 2016 update. MLTJ. 2016;6(1):1-5.

9. Thomee R, Renstrom P, Karlsson J, Grimby G. Patellofemora pain syndrome in young women. I. A clinical analysis of alignment, pain parameters, common symptoms and functional activity level. Scand J Med Sci Sports. 1995;5:237-244.

10. Witvrouw E, Sneyers C, Lysens R, Victor J, Bellemans J. Reflex response times of vastus medialis oblique and vastus lateralis in normal subjects and in subjects with patellofemoral pain syndrome. J Orthop Sports Phys Ther. 1996;24:160-165.

11. Laprade J, Culham E, Brouwer B. Comparison of five isometric exercises in the recruitment of the vastus medialis oblique in persons with and without patellofemoral pain syndrome. J Orthop Sports Phys Ther. 1998;27:197-204.

12. Jensen MP, Turner JA, Romano JM. What is the maximum number of levels needed in pain intensity measurement? Pain. 1994;58:387-392.

13. Kujala UM, Jaakkola LH, Koskinen SK, Taimela S, Hurme M, Nelimarkka O. Scoring of patellofemoral disorders. Arthroscopy. 1993;9:159-163.

14. Cowan SM, Bennell KL, Hodges PW, Crossley KM, McConnell J. Delayed onset of electromyographic activity of vastus lateralis compared to vastus medialis obliquus in subjects with patellofemoral pain syndrome. Arch Phys Med Rehabil. 2001;82:183-189.

15. Dvir Z, Halperin N, Shklar A, Robinson D. Quadriceps function and patellofemoral pain syndrome. Part I: Pain provocation during concentric and eccentric isokinetic activity. Isokinetics and Exercise Science. 1991;11:26-30.

16. Kaya D, Atay OA, Callaghan MJ, Cil A, Caglar O, Citaker S, Yuksel I, Doral MN. Hallux valgus in patients with patellofemoral pain syndrome. Knee Surg Sports Traumatol Arthrosc. 2009;17:1364-1367.

17. Nordin M, Frankel V. Basic Biomechanics of the Musculoskeletal System, London, England: Lippincott Williams \& Wilkins. 1989.

18. Foti C, Laurini A, Tiberti S, Carli G, Tsarpela O, Adamidis K, Bonifazi M, Giombini A, Tihanyi J, von Duvillard S, De Vita M, Bosco C. Leg extension test, sEMG and vibratory stimuli to assess functional recovery following knee joint surgery. Muscles Ligaments Tendons Journal. 2012; 2: 127-132.

19. Kaya D, Doral MN, Callaghan M. How can we strengthen the quadriceps femoris in patients with patellofemoral pain syndrome? Muscles Ligaments Tendons Journal. 2012; 2: 25-32. 